\title{
Neue Annäherungen an Stadt und Land
}

\author{
Andreas Klee · Gregor Prinzensing
}

Online publiziert: 22. Juli 2011

(C) Springer-Verlag 2011

In Heft 2.2011 von „Raumforschung und Raumordnung“ erschienen zwei Beiträge mit einem gemeinsamen Ursprung: Sie sind aus einer Fachsitzung beim Deutschen Geographentag 2009 in Wien hervorgegangen. Diese Sitzung mit dem Titel „Die Rückkehr des Urbanen?“ wurde veranstaltet, um empirische Studien und theoretisch-konzeptionelle Überlegungen zu Stadtregionen im Kontext der Fragestellung zu diskutieren, inwiefern die Kernstädte nach Jahrzehnten des Bedeutungsverlusts wieder an Bedeutung gewinnen. In ihrem gleichnamigen Gast-Editorial stellten Christina West und Sebastian Lentz im April 2011 in Aussicht, es würden weitere Arbeiten in künftigen Ausgaben von „Raumforschung und Raumordnung“ folgen, die die Dynamik in dieser sowohl empirisch gestützten wie auch theoretisch inspirierten Diskussion um den Forschungsgegenstand „Stadt“ und sein Forschungsfeld zeigen.

Mit der nun vorgelegten Ausgabe von ,Raumforschung und Raumordnung“"veröffentlichen wir einen weiteren Beitrag aus diesem Umkreis. Der Artikel von Anne Vogelpohl „Städte und die beginnende Urbanisierung“ diskutiert die Bedeutung der Theorien von Henri Lefebvre für die aktuelle Stadtforschung und knüpft dazu an seine Konzeption von der Produktion des Raumes an. Das zentrale Theorem von der „Urbanisierung der Gesellschaft" wird reformuliert und der Unterschied zwischen den Vokabeln „Stadt“ und „Urba-

Dr. A. Klee $(\triangle)$

Akademie für Raumforschung und Landesplanung -

Leibniz-Forum für Raumwissenschaften, Hohenzollernstraße 11,

30161 Hannover, Deutschland

E-Mail: Klee@ARL-net.de

G. Prinzensing

Leibniz-Institut für Regionalentwicklung und Strukturplanung,

Flakenstraße 28-31, 15537 Erkner, Deutschland

E-Mail: prinzensing@irs-net.de nes“ herausgearbeitet. Die „Stadt“ im Sinne Lefebvres ist als eine historische Bedingung zu verstehen, die im Wesentlichen mit der Industrialisierung untergegangen ist. Mit dem „Urbanen“ wird hingegen eine künftige, auf Differenzen basierende Gesellschaftsformation bezeichnet, die bis jetzt weitgehend virtuell existiert.

Einem aktuellen raumentwicklungspolitischen Ansatz widmet sich in diesem Heft Sandra Pennekamp mit einer empirischen Untersuchung $\mathrm{zu}$ überregionalen Partnerschaften. Ihre Arbeit bezieht sich auf das gleichnamige Modellvorhaben der Raumordnung, das zum Ziel hatte aufzuzeigen, wie überregionale Kooperationen organisiert werden können und wie dadurch ein Beitrag geleistet werden kann, Wachstum und Innovation zu erzeugen. Können die Partnerschaften das leisten? Sandra Pennekamp hat hierzu vier Regionen untersucht. Sie macht deutlich, dass zum Gelingen einer solchen Partnerschaft eine Reihe von Voraussetzungen notwendig sind. Und sie macht deutlich, dass neben den finanziellen und personellen Ressourcen vor allem die Motivation der beteiligten Akteure, getragen von partnerschaftlichem Engagement, Vertrauen und Gestaltungswillen, eine entscheidende Stellgröße für das „Funktionieren" einer überregionalen Partnerschaft und letztlich auch für eine erfolgreiche Regional Governance darstellt.

Regional Governance spielt auch beim Hochwasserschutz eine entscheidende Rolle. Dies stellt Thomas Hartmann in seinem Bericht „Den Flüssen mehr Raum geben - Umsetzungsrestriktionen in Recht und Praxis“" heraus. Die bestehenden rechtlichen Instrumentarien von Wasserwirtschaft und räumlicher Planung zum vorbeugenden Hochwasserschutz reichen seiner Ansicht nach nicht aus, ein flächenorientiertes Risikomanagement zu gewährleisten. Er kommt zum Schluss, dass ein Umdenken erfolgen muss. Die Umsetzung der rechtlich vorgegebenen Instrumentarien in der Praxis benötigt neue Formen von Regional Gover- 
nance, nämlich integrative Planungsprozesse, in die neben Akteure der Wasserwirtschaft auch die der räumlichen Planung sowie Grundstückseigentümer einzubinden sind. Thomas Hartmann hat hierzu mit Hilfe einer Politikfeldanalyse die Hochwasserkonzeption des Landes Sachsen-Anhalt und deren Umsetzung vor und nach dem Inkrafttreten des Hochwasserschutzgesetzes von 2005 untersucht.

Einem anderen Planungsproblem widmen sich Robert Wieser und Wilfried Schönbäck in ihrem Bericht „Volkswirtschaftliche und raumordnungspolitische Aspekte der Widmungsabgabe nach dem Entwurf zur Novelle zum Tiroler Raumordnungsgesetz". Die Autoren stellen sich die Frage, wie der in vielen Tiroler Gemeinden anzutreffende hohe Baulandüberhang einer tatsächlichen Bebauung zugeführt werden kann, um dadurch neue Flächenwidmungen zu vermeiden. Im Rahmen der Novelle des Tiroler Raumordnungsgesetzes wurde in diesem Zusammenhang die Erhebung einer „Widmungsabgabe“ in jenen Fällen, in denen sich durch Umwidmung von Grundstücken im Vergleich zur vorherigen Widmung eine Steigerung des ortsüblichen Verkehrswertes ergibt, diskutiert. Die Widmungsabgabe wurde zwar nicht realisiert. Gleichwohl zeigen Robert Wieser und Wilfried Schönbäck auf, welche bodenökonomischen und finanziellen Wirkungen diese Abgabe hätte entfalten können.

Wir hoffen, dass wir mit diesen Beiträgen und Berichten wieder einen Querschnitt der aktuellen raumbezogenen Forschung abbilden können und wünschen den Leserinnen und Lesern von „Raumforschung und Raumordnung“ neue Anregungen und Erkenntnisse. 